\title{
Seguimiento de diferentes tipos de corrosión mediante la aplicación de la transformada de wavelets a registros de ruido en corriente ${ }^{(\cdot)}$
}

\author{
Álvaro Aballe*, Manuel Bethencourt*, \\ Francisco Javier Botana* y Mariano Marcos*
}

\begin{abstract}
Resumen La dificultad más importante para usar la medida del ruido electroquímico reside en la interpretación de los datos experimentales. En la literatura, se han propuesto básicamente tres aproximaciones diferentes para procesar los registros experimentales: la estadística, la espectral y los métodos basados en la teoría del caos. El propósito de este trabajo es introducir una herramienta alternativa para el análisis del ruido electroquímico: la transformada de wavelets. Se ha encontrado que esta herramienta podría ser especialmente útil para distinguir procesos localizados y uniformes que se desarrollan simultáneamente. Con este objetivo, se han analizado mediante la transformada de wavelets registros de ruido de corriente correspondientes a electrodos de trabajo que sufren ambos tipos de procesos de corrosión. Estos resultados son comparados con los obtenidos al aplicar la transformada de Fourier. Además, se presenta brevemente una descripción teórica de la herramienta y se sugieren algunas aplicaciones en el campo del control de la corrosión industrial.
\end{abstract}

Palabras Claves Ruido electroquímico. Wavelets. Corrosión. Análisis de datos. Vigilancia. Tipos de corrosión.

\section{Monitoring of different corrosion types through the application of wavelet transform to current noise records}

\begin{abstract}
The most important difficulty to use electrochemical noise measurement resides in the interpretation of the experimental data. In the literature, three different main approaches have been proposed for processing the experimental records: the statistical, the spectral and the Chaos-Theory based methods. The purpose of this work is to introduce an alternative tool to analyse electrochemical noise: the wavelet transform. It has been found that this tool could be especially usefully to distinguish localised and uniform corrosion processes that are developing simultaneously. To show that, a current noise record corresponding to working electrodes suffering from both kind of corrosion process is analysed by means of the wavelet transform. These results are compared with the ones coming form the Fourier. In addition, a brief theoretical description of the tool is presented and some applications in the field of the industrial monitoring are suggested.
\end{abstract}

Keywords Electrochemical noise. Wavelets. Corrosion. Data processing. Monitoring. Type of corrosion.

\section{INTRODUCCIÓN}

La Medida del Ruido Electroquímico (ENM) es una técnica electroquímica cuya aplicación en el seguimiento y control de procesos de corrosión se ha incrementado enormemente en los últimos años. Fundamentalmente, este incremento se debe al carácter no intrusivo del proceso de medida y al bajo coste de la instrumentación requerida para llevarla a cabo. Para realizar este tipo de medidas el procedimiento experimental más habitual consiste en el registro simultáneo de las variaciones temporales de la corriente que fluye entre dos electrodos nominalmente idénticos conectados entre sí y las variaciones de diferencia de potencial entre dicho par y un electrodo de referencia. Las señales obtenidas constituyen lo que se conoce como ruido electroquímico. Con frecuencia, la interpretación de los resultados experimentales no es evidente y se hace imprescindible un tratamiento matemático de

(•) Trabajo recibido el día 17 de octubre de 1999 y aceptado en su forma final el día 3 de diciembre de 1999.

(*) Departamento de Ciencia de los Materiales e Ingeniería Metalúrgica y Química Inorgánica. Universidad de Cádiz. Facultad de Ciencias del Mar. Apartado 40.11510 Puerto Real. Cádiz (España). 
los datos. El análisis matemático de los Registros de Ruido Electroquímico (ENR) puede proporcionar información acerca de la velocidad y mecanismo de los procesos de corrosión que tienen lugar en el sistema estudiado ${ }^{[1]}$. En la bibliografía se encuentran recogidos, básicamente, tres tipos de métodos diferentes para el tratamiento de datos procedentes de la ENM. Así, por un lado, el método estadístico se basa, fundamentalmente, en el análisis de la dispersión de los valores experimentales ${ }^{[2-3]}$. Mediante este método se ha podido definir una magnitud denominada resistencia de ruido, que se relaciona con la resistencia de polarización, a partir de la cual puede determinarse la velocidad de corrosión del sistema $^{[4]}$. Por otro lado, el método espectral efectúa un análisis en el dominio de frecuencias de los registros de ruido electroquímico $\mathrm{ENR}^{[5-6]}$. Para este tipo de estudios se emplea el algoritmo de transformada rápida de Fourier (FFT) o el método de la máxima entropía (MEM) ${ }^{[7]}$. Mediante este tipo de análisis es posible extraer información acerca de la constante de tiempo de los tránsitos que forman un registro y de la frecuencia con que estos ocurren ${ }^{[8]}$. Finalmente, un tercer método de análisis se basa en la aplicación de conceptos relacionados con la teoría del caos ${ }^{[1,9]}$ y su principal aplicación reside en la identificación del tipo de proceso de corrosión predominante.

Sin embargo, los métodos matemáticos anteriormente descritos encuentran serias limitaciones, especialmente, cuando el sistema que se estudia se ve afectado por la superposición de diferentes procesos de corrosión que ocurren de forma casi simultánea. En estos casos, el registro temporal completo resulta de la composición de las señales elementales correspondientes. Así, al utilizar parámetros estadísticos, como la desviación estándar, se consideran al mismo tiempo todas las componentes de la señal con lo cual es imposible evaluar la contribución de cada proceso. Por lo que se refiere al análisis de Fourier, es preciso señalar que este método sólo es aplicable a señales estacionarias. Por esta razón, los registros de ruido electroquímico que contengan tránsitos o derivas no deberían ser analizados mediante transformada de Fourier. Finalmente, si bien es posible distinguir el tipo de proceso de corrosión predominante usando relaciones basadas en la teoría del caos, este método difícilmente permite evaluar el peso relativo de cada componente.

En el presente trabajo se propone un método alternativo para el análisis de los datos experimentales obtenidos mediante la ENM, que permita sal- var los inconvenientes anteriormente citados. Dicho método se basa en la aplicación de la transformada de wavelets en el tratamiento de los registros de ruido electroquímico.

\section{TRANSFORMADA DE WAVELETS: FUNDA- MENTO TEÓRICO}

El análisis de datos mediante el uso de wavelets es una herramienta matemática de desarrollo reciente que se plantea como una alternativa al análisis mediante transformada de Fourier. A continuación se presentan brevemente los fundamentos matemáticos de este tipo de transformada. En ${ }^{[10]}$ se describen con mayor detalle los procedimientos empleados en este trabajo.

Análogamente a lo que ocurre con las transformadas de Fourier, se han desarrollado dos clases de transformadas de wavelets, denominadas continua (CWT) y discreta (DWT). Dentro de estas últimas, se han diferenciado distintas transformadas de wavelets. Entre ellas se encuentra la denominada trasformada de wavelets ortogonales (OWT). El algoritmo utilizado para llevar a cabo el cálculo depende del tipo de transformada (CWT, DWT, OWT) que se desee realizar. Las consideraciones teóricas recogidas en este apartado se refieren a las transformadas del tipo OWT.

Consideremos un registro temporal $x_{d}(d=$ 1 ...N). La aproximación esencial de wavelets consiste en representar el registro temporal $x_{d}$ usando una combinación lineal de funciones bases $\phi_{j, n} \mathrm{Y}$ $\psi_{j, n}{ }^{[11]}$ :

$$
x(t) \approx \sum_{n} s_{J, n} \phi_{J, n}(t)+\sum_{n} d_{J, n} \Psi_{J-1, n}(t)+\ldots+\sum_{n} d_{1, n} \Psi_{1, n}(t)
$$

donde $s_{\mathrm{J}, \mathrm{n}}, d_{\mathrm{J}, \mathrm{n}}, \ldots, d_{1, \mathrm{n}}$ son los denominados coeficientes de wavelets. Los valores de estos coeficientes se obtienen, aproximadamente, a partir de las siguientes expresiones:

$$
\begin{aligned}
s_{J, n} & \approx \int x(t) \phi_{J, n}(t) d t \\
d_{j, n} & \approx \int x(t) \psi_{j, n}(t) d t
\end{aligned}
$$

Las funciones base de wavelets $\phi_{\mathrm{j}, \mathrm{n}} \mathrm{y} \psi_{\mathrm{j}, \mathrm{n}}$ se generan a partir de una pareja de funciones, denominadas wavelets padre, $\phi$, y wavelets madre, $\psi$, utilizando para ello procesos de escala y traslación, de acuerdo con las expresiones

$$
\phi_{j, n}(t)=2^{-j / 2} \phi\left(2^{-j} t-n\right)=2^{-j / 2} \phi\left(\frac{t-2^{j} n}{2^{j}}\right)
$$




$$
\psi_{j, n}(t)=2^{-j / 2} \psi\left(2^{-j} t-n\right)=2^{-j / 2} \psi\left(\frac{t-2^{j} n}{2^{j}}\right)
$$

en las que $n=1,2, \ldots N / 2^{\mathrm{j}}$ y $j=1,2, \ldots, J$, siendo $J$ un número natural suficientemente pequeño. Dicho número depende, principalmente, de $\mathrm{N}$ y las características de $\phi, \psi$. En la figura 1 se han representado algunos ejemplos de bases de wavelets con distintos valores de $j$ y $n$. Estas bases se definen en todo el intervalo del eje de abscisas, si bien, sus valores sólo son diferentes de cero en un intervalo determinado. La posición de este intervalo depende de $n$, mientras que $j$ determina la amplitud de dicho intervalo. Una función base con un coeficiente $j$ difiere de otra con coeficiente $j-1$ en que la primera ha sido obtenida a partir de la segunda aplicando un proceso de estiramiento horizontal y otro de encogimiento vertical. Por lo tanto, además de verificarse las ecuaciones (4) y (5), deben ser satisfechas las siguientes:

$$
\begin{aligned}
& \int \phi_{J, n}(t) d t=1 \\
& \int \psi_{j, n}(t) d t=0
\end{aligned}
$$

Los coeficientes de wavelets miden el grado de correlación entre las funciones base de wavelets, incluyendo sus picos y valles, y la señal estudia$\mathrm{da}^{[12]}$. De acuerdo con las ecuaciones (2) y (3), esta correlación se mide a partir del área entre el eje de abscisas y la curva resultante al multiplicar la

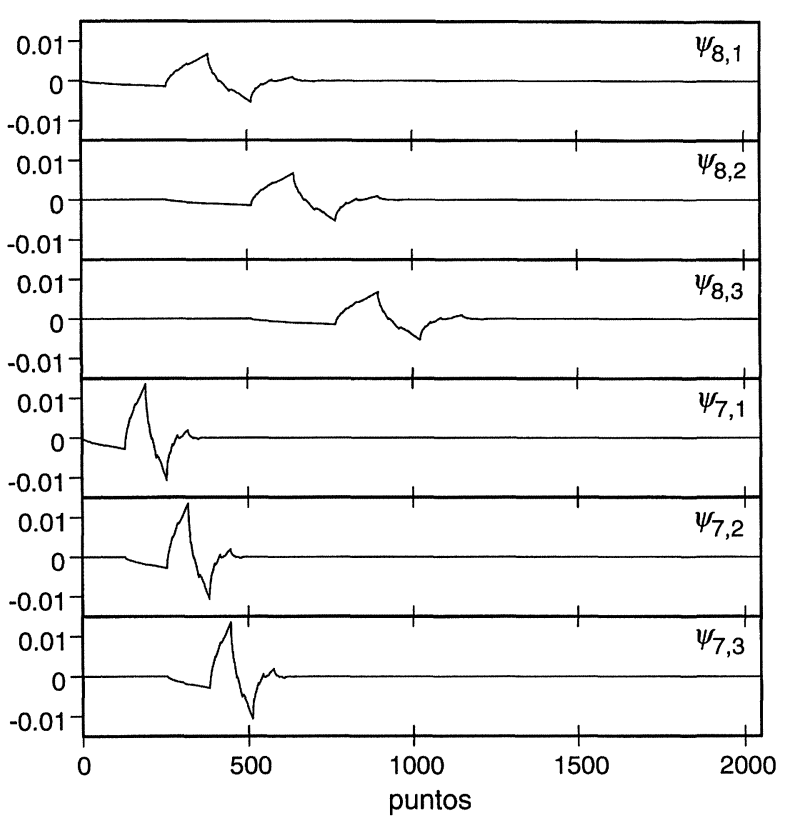

Figura 1. Ejemplos de bases de wavelets.

Figure 1. Examples of wavelet basis. señal por la correspondiente función base. Para ilustrar este procedimiento, en la figura 2 se ha representado una señal sinusoidal $D(t)$ junto con el producto de esta señal por dos funciones base de wavelets diferentes. La diferencia entre estos dos productos es debida a que la amplitud y frecuencia de $D(t)$ cambian a lo largo del eje de abscisas. Así, las áreas contenidas en las partes positivas y negativas de $D(t) \cdot \psi_{7,1}$ se cancelan mutuamente, de manera que el área resultante es muy pequeña. Esto implica que el coeficiente de wavelets correspondiente también tenga un valor pequeño.

Por otro lado, en el intervalo en el que $\psi_{7,4}$ no es nulo, las mayores amplitudes de $D(t)$ y la mayor coincidencia en forma de la función base y $D(t)$ en este intervalo, hacen que el área bajo la curva $D(t) \cdot \psi_{7,4}$ sea considerablemente mayor. Como consecuencia, el coeficiente de wavelets correspondiente presentará un valor mayor. En definitiva, ya que sólo en un intervalo de tiempo las funciones basé no son nulas, un coeficiente de wavelets informa solamente sobre un determinado segmento de la señal original, dando un valor mayor cuanto mayor sea la amplitud de la señal estudiada y su nivel de coincidencia con la función base. Así, la señal puede ser estudiada segmento a segmento variando el valor de $n$ de la base de wavelets. Por otra parte, como se puede observar en la figura 1, es posible descomponer la señal original en componentes con diferentes escalas variando el valor de $j$ de las funciones bases.

En resumen, mediante la transformada de wavelets es posible descomponer el registro original en señales diferentes y cuantificar la aportación individual de cada una de estas señales al registro completo.

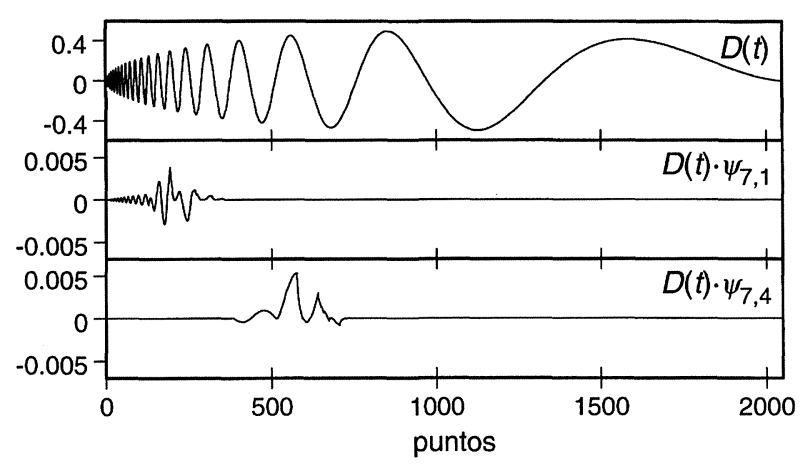

Figura 2. Ejemplos de productos de bases de wavelets por una señal doppler $D(t)$.

Figure 2. Examples of multiplication of wavelets basis by a doppler signal $D(t)$. 


\section{PARTE EXPERIMENTAL}

Los datos de ruido electroquímico incluidos en este trabajo corresponden al sistema formado por probetas de acero AISI 304 sumergidas en disolución $\mathrm{FeCl}_{3}$ 0,001M. Con anterioridad a los ensayos, se pulieron las probetas hasta un grado de 500 -grits usando papel de $\mathrm{SiC}$, seguidamente se enjuagaron con agua destilada y, finalmente, se desengrasaron con acetona. Para realizar los ensayos de ENM, se usaron dos electrodos de $1 \mathrm{~cm}^{2}$, los cuales se colocan de forma paralela dentro de una celda. Los datos se registraron a una velocidad de muestreo de 2,15 puntos por segundo hasta completar 2.048 puntos. De esta forma las señales de ruido de corriente al potencial de corrosión se registran con una resolución de $1 \mathrm{pA}$, utilizando para ello una interfase electroquímica 1287SI de Solartron.

Para realizar las transformadas de wavelets en los ENR, se diseñó una rutina específica de análisis de datos usando el módulo $\mathrm{S}+$ Wavelets de la aplicación S-PLUS. Esta rutina incluye la eliminación de la media de los ENR previamente de la aplicación de la transformada de wavelets. Para la realización de esta transformación es necesario definir las correspondientes condiciones de contorno y las wavelets padre y madre, $\phi$ y $\psi$. Los resultados de este trabajo han sido obtenidos imponiendo unas condiciones de contorno en las cuales las funciones bases son anuladas fuera del rango de tiempo del ENR ${ }^{[13]}$. Así, es posible analizar los datos sin necesidad de eliminar las posibles derivas antes de realizar la transformada de wavelets, a diferencia de lo que ocurre para el análisis de Fourier. Las condiciones de contorno seleccionadas limitan las wavelets padre y madre, $\phi$ y $\psi$ que se pueden utilizar. En este caso, sólo pueden ser utilizadas las denominadas wavelets symmlets ${ }^{[14]}$. En la figura 1 se muestran algunas de las bases de wavelets obtenidas a partir de la wavelet madre empleada en el desarrollo del presente trabajo.

\section{RESULTADOS Y DISCUSIÓN}

\subsection{Análisis de los coeficientes de wavelets}

Si se realiza la transformada de wavelets bajo las condiciones anteriormente descritas, se obtienen nueve $(J=8)$ grupos de coeficientes de wavelets denominados cristales:

$$
\begin{gathered}
d_{1}=\left(d_{1,1}, d_{1,2}, \ldots, d_{1,1024}\right), d_{2}=\left(d_{2,1}, d_{2,2}, \ldots, d_{2,512}\right) \ldots \\
d_{8}=\left(d_{8,1}, d_{8,2}, \ldots, d_{8,8}\right), s_{8}=\left(s_{8,1}, s_{8,2}, \ldots, s_{8,8}\right)
\end{gathered}
$$

Cada cristal ofrece una descripción de la señal a una escala de tiempo determinada. De manera que esta escala será mayor cuanto mayor sea el intervalo no nulo de las bases correspondientes al cristal. Los intervalos de escala correspondiente a los cristales $d_{1}-d_{7}$ pueden ser estimados, aproximadamente, a partir de la ecuación

$$
\left(C_{1}^{j}, C_{2}^{j}\right)=\left(2^{j} \Delta t, 2^{j-1} \Delta t\right)
$$

donde $\mathrm{C}_{1}^{\mathrm{j}}$ y $\mathrm{C}_{2}^{\mathrm{j}}$ son, respectivamente, el principio y el final del intervalo de la escala; $\Delta t$ es el intervalo de muestreo; y $j$ representa el nombre del cristal correspondiente ${ }^{[11]}$. En cuanto al cristal $\mathrm{s}_{8}$, es el que contiene información sobre procesos que tiene lugar con una escala de tiempo mayor que $C_{2}^{J}$.

En la figura 3 se han representado los coeficientes de wavelets de una señal de ruido de corriente. La señal original, que aparece en la parte superior de la figura, corresponde a una muestra de acero inoxidable' 304 sumergido durante $10 \mathrm{~h}$ en una disolución de $\mathrm{FeCl}_{3}$ 0,001M. Los coeficientes de wavelets están representados en las filas restantes, partiendo de los de escala más fina (cristal $d_{1}$ ), en la segunda fila, a los coeficientes de escala más gruesa (cristal $s_{8}$ ) en la fila inferior.

Cada cristal está formado por coeficientes que, como se explicó con anterioridad, proporcionan información de un segmento de la señal con una determinada longitud. Estos coeficientes están representados como líneas verticales en el centro aproximado de cada segmento. Los coeficientes $d_{1}$ a $d_{8}$ han sido representados a la misma escala mientras que la señal original y el cristal $s_{8}$ lo han sido a una escala vertical diferente porque presentan valores mucho mayores. Obsérvese que el cristal $d_{\mathrm{j}}$ contiene la mitad de coeficientes que $d_{\mathrm{j}-1}$ como consecuencia de la mayor longitud del intervalo en el que la base correspondiente contiene valores no nulos. Esto significa que la resolución con la que se analiza la señal disminuye a la mitad al aumentar $j$.

El ENR de la parte superior de la figura 3 se caracteriza por presentar tres tránsitos que destacan sobre otras fluctuaciones de alta frecuencia y baja amplitud. Por lo tanto se puede considerar que el registro está formado por dos componentes superpuestas. Cada una de estas componentes puede estar relacionada con procesos corrosivos de diferente naturaleza que actúan simultáneamente. Uno de ellos podría relacionarse con un proceso de corrosión localizada. Dicho proceso produce una señal que, habitualmente, se caracteriza por contener 


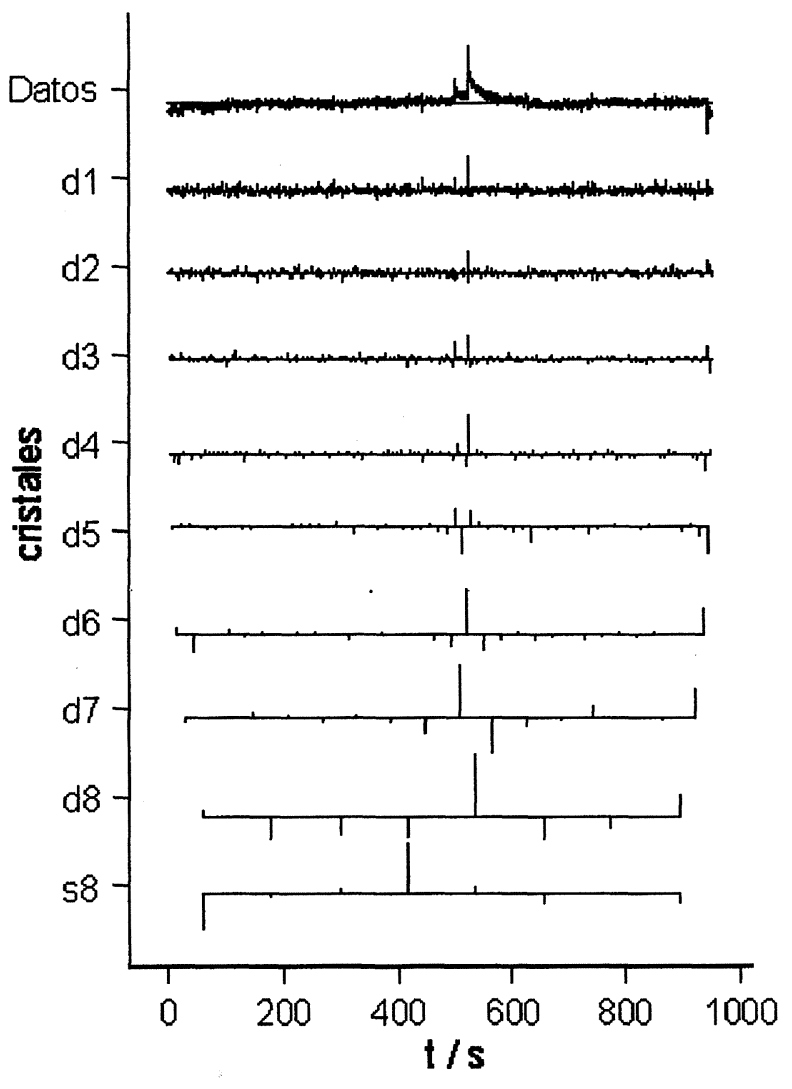

Figura 3. Coeficientes de wavelets provenientes de una transformada de wavelets de un registro de ruido de corriente correspondiente a una muestra de acero inoxidable 304 después de 7 horas en una disolución de $\mathrm{FeCl}_{3} 0.001 \mathrm{M}$.

Figure 3. Wavelet coefficients resulting form wavelet transform of a current noise record corresponding to a sample of $304 \mathrm{SS}$ after 7 hours in a $0.001 \mathrm{M} \mathrm{FeCl}_{3}$ solution.

una serie de tránsitos con una forma característica, como los que aparecen en la figura $3^{[15]}$. La segunda componente podría asociarse a un proceso de corrosión uniforme, caracterizado por una señal formada por fluctuaciones rápidas que presentan una distribución aproximadamente normal.

Obsérvese que las fluctuaciones correspondientes al proceso de corrosión uniforme aparecen a lo largo de toda la señal experimental. Esto hace que los coeficientes de wavelets asociados a ellas tengan valores semejantes y distintos de cero en todos los segmentos. Por otra parte, en cada cristal los mayores coeficientes son atribuibles, por su posición, a los tránsitos que aparecen en la señal experimental. La forma de estos tránsitos hace que se reflejen en todos los cristales de manera que su existencia se detecta por la repetición en la misma posición de coeficientes con valores mucho más altos en todos o casi todos los cristales. Esta propiedad puede ser utilizada para automatizar la detección de tránsitos asociados a procesos de corrosión localizada.
Aunque los tránsitos quedan reflejados en todos los cristales, su posición exacta en el tiempo debe ser medida en el cristal de mayor resolución, $d_{1}$.

\subsection{Diagramas de distribución de energía}

Una forma alternativa de presentar el resultado de la transformada de wavelets puede ser la estimación de la contribución de cada cristal sobre el total de la señal. En este contexto, la energía de la señal puede ser calculada de acuerdo a la siguiente ecuación:

$$
E=\sum_{d=1}^{N} x_{d}^{2} \quad d=1, \ldots, N
$$

Entonces, la fracción de energía asociada con cada cristal se calcularía:

$$
\begin{gathered}
E_{j}^{d}=\frac{1}{E} \sum_{n=1}^{N / 2^{j}} d_{j, n}^{2} j=1, \ldots, J \\
E_{j}^{s}=\frac{1}{E} \sum_{n=1}^{N / 2^{8}} s_{J, n}^{2}
\end{gathered}
$$

La representación de la energía relativa acumulada por cada cristal frente al nombre del cristal, $j$, será denominada de aquí en adelante como diagrama de distribución de energía (EDP). En la figura 4 se muestra el EDP correspondiente a la figura 3. Existen dos máximos que aparecen en los cristales $d_{1}$ y $s_{8}$. Así, estos máximos indicarían la presencia de dos componentes que actúan a diferente escala de tiempo. De acuerdo con lo dicho en el apartado anterior, los coeficientes correspondientes a pequeña escala estarían relacionados con un proceso

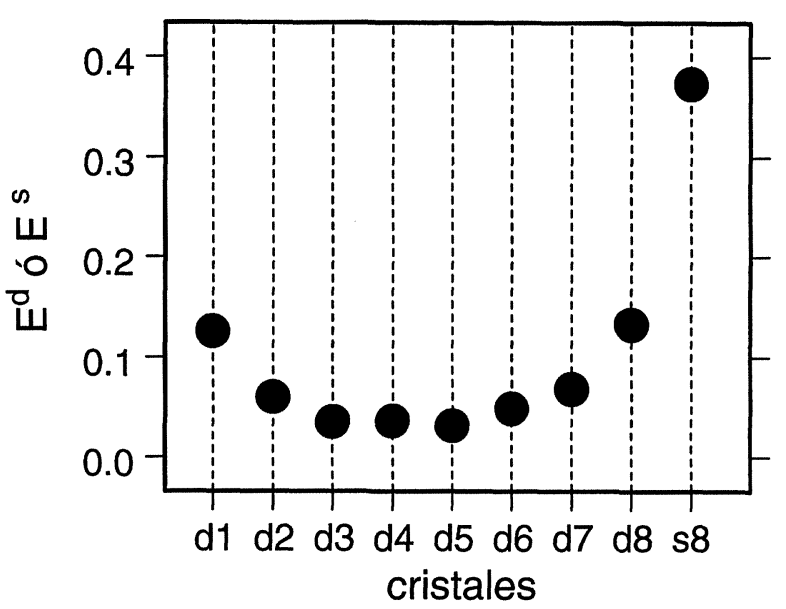

Figura 4. Diagrama de distribución de energía correspondiente a la Figura 3.

Figure 4. Energy distribution plot corresponding to Figure 3. 
de corrosión uniforme mientras que los cristales de gran escala lo estarían con un proceso de corrosión localizada. Si bien es cierto que es posible distinguir los dos procesos directamente mediante la simple observación del registro temporal, la transformada de wavelets puede ser útil para automatizar aplicaciones de seguimiento y control de la corrosión en la industria o para cuantificar la importancia relativa de cada proceso individual.

Este tipo de representación puede ser usado como una especie de "huella dactilar" de la señal, de una forma parecida a los espectros de densidad de potencia (PSD). Sin embargo, es importante enfatizar que la transformada de wavelets parece ser más apropiada para el estudio de los registros de ENM. En la figura 5 se muestra el PSD correspondiente a la señal experimental incluida en la figura 3 . Obsérvese que es difícil distinguir la existencia de dos procesos a partir de esta figura e incluso más, extraer alguna información cuantitativa. Por tanto, cabe destacar que la transformada de wavelets, especialmente diseñada para el análisis de tránsitos en una señal, puede constituir un método alternativo de gran utilidad para el análisis de los datos experimentales obtenidos mediante la medida del ruido electroquímico, superándose los inconvenientes inherentes al análisis de Fourier, que no ofrece buenos resultados cuando la señal estudiada es no estacionaria, caso frecuente en el ruido electroquímico.

A continuación se presentan los resultados obtenidos al aplicar la metodología propuesta en párrafos anteriores al análisis de los registros correspondientes a un acero inoxidable 304 en disolución de $\mathrm{FeCl}_{3}$ $0,001 \mathrm{M}$. Adicionalmente, se propone una sistemática que permite seguir la evolución en el tiempo de los dos tipos de procesos detectados en el estudio de

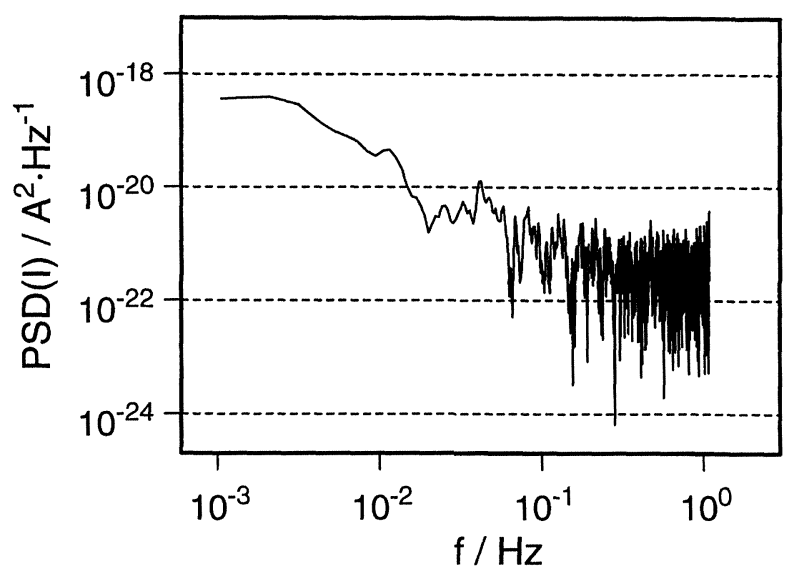

Figura 5. Espectro de densidad de potencia correspondiente al registro temporal de la Figura 3.

Figure 5. Power spectral density corresponding to the current noise record in Figure 3. dicho material. Para ello, en primer lugar, sería necesario determinar en qué cristales se refleja principalmente cada tipo de proceso. Con este objetivo se han elegido dos ENR en los que existe un claro predominio de cada uno de los procesos. Los registros seleccionados han sido los correspondientes a ensayos realizados a tiempos de inmersión de 2 y $48 \mathrm{~h}$ (Fig. 6). En la figura 6(a) puede observarse la existencia de tránsitos característicos de un ataque localizado. Estos tránsitos tienen una amplitud mucho mayor que los existentes en la figura 3, lo que indicaría una mayor intensidad del proceso de ataque localizado. Por otra parte, los tránsitos en la figura 6(b) se confunden con las oscilaciones debidas al proceso de corrosión uniforme, lo cual vendría a indicar que, en estas condiciones, el sistema está dominado por el proceso de corrosión uniforme. Por lo tanto, los EDPs correspondientes a estos registros (Fig. 7), pueden servir para indicarnos en qué cristales se manifiesta cada proceso. De la figura 7 se deduce que el proceso de corrosión localizada se caracteriza por presentar elevados valores de energía relativa alrededor del cristal $d_{7}$. Por otra parte, la energía relativa asociada al proceso de corrosión uniforme se concentra en los cristales de $j$ más bajo. A partir de esta asignación, se podría sugerir un procedimiento que permitiera efectuar un seguimiento temporal de cada tipo de proceso de forma individualizada. Para ello, sería necesario calcular la variación, en el tiempo, de las sumas de la energías relativas de los cristales $d_{8}$ $d_{6}$, para el proceso localizado, y la de los cristales $d_{3}$ $d_{1}$, para el uniforme. Se ha evitado considerar el cristal $s_{8}$ porque en él quedan incluidos los efectos de la posible deriva de la señal.

En la figura 8 se ha representado el resultado de aplicar el procedimiento anteriormente descrito sobre registros de ruido en corriente adquiridos a

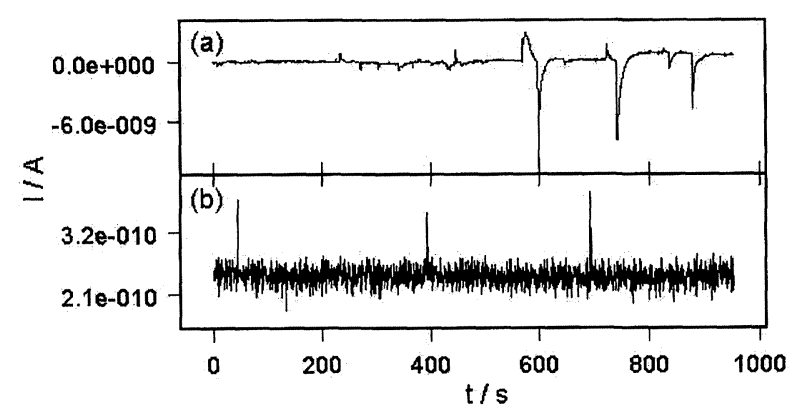

Figura 6. Registros de ruido en corriente correspondientes a muestras de acero inoxidable 304 después de $2 \mathrm{~h}$ (a) y $48 \mathrm{~h}$ (b) en una disolución de $\mathrm{FeCl}_{3}$ 0,001M.

Figure 6. Current noise recording corresponding to 304 stainless steel samples after $2 \mathrm{~h}(\mathrm{a})$ and $48 \mathrm{~h}(\mathrm{~b})$ in $0.001 \mathrm{M}$ $\mathrm{FeCl}_{3}$ solution. 


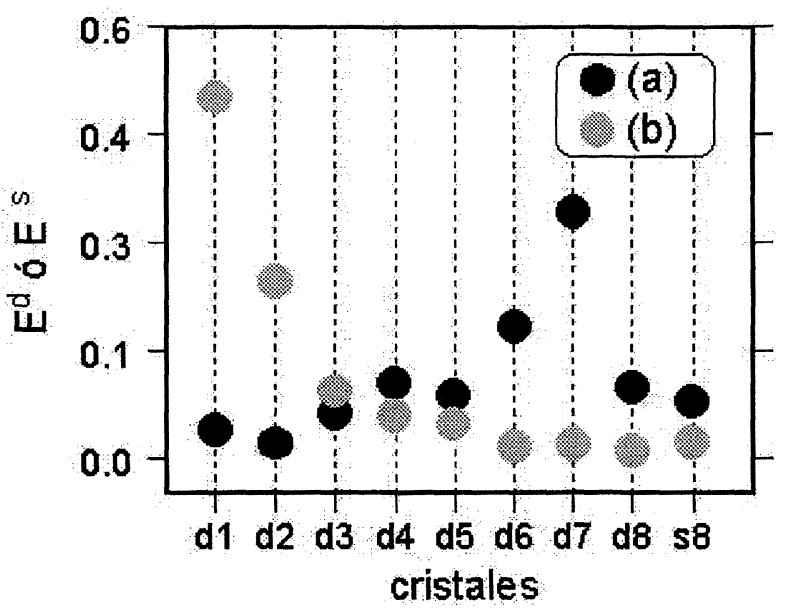

Figura 7. Diagramas de distribución de energía correspondientes a los registros incluidos en la figura 6 .

Figure 7. Energy distribution plots corresponding to the records included in figure 6.

intervalos de una hora para el sistema estudiado. Esta representación nos permite determinar la existencia de tres etapas. Así, durante las primeras $9 \mathrm{~h}$ se observa un claro predominio del proceso localizado; durante las siguientes $10 \mathrm{~h}$ el proceso localizado se da de forma intermitente; y, finalmente, sólo se observa proceso de corrosión uniforme de baja intensidad.

\section{CONCLUSIONES}

En este trabajo se propone el empleo de la transformada de wavelets como método alternativo en el análisis de registros de ruido electroquímico. Para ello, primeramente, se ha descrito brevemen-

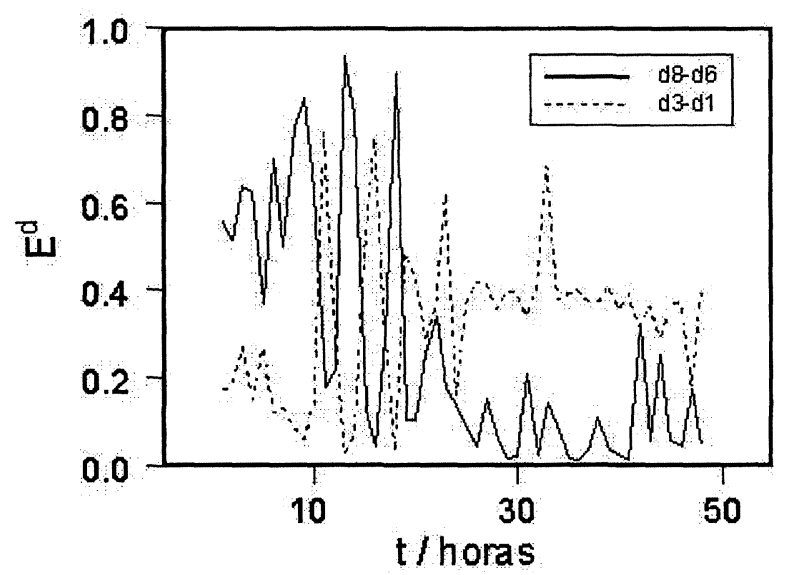

Figura 8. Fracción de energía acumulada por los cristales $(d 8-d 6)$ y $(d 3-d 1)$ en función del tiempo de exposición de muestras de acero inoxidable 304 en una disolución de $\mathrm{FeCl}_{3} 0,001 \mathrm{M}$.

Figure 8. Energy fraction gathered by the $\left(d_{8}-d_{6}\right)$ and $\left(d_{3}-d_{1}\right)$ crystals versus the exposure time for 304 stainless steel samples in a $0.001 \mathrm{M} \mathrm{FeCl}_{3}$ solution. te los fundamentos de la transformada de wavelets. A continuación, se han propuesto dos formas diferentes de presentación de resultados, los cuales han sido comparados con los provenientes de un análisis de Fourier clásico. Finalmente, a modo de ejemplo, se ha aplicado la sistemática propuesta en el análisis de una serie de registros experimentales de ruido en corriente.

Los resultados obtenidos ponen de manifiesto que al aplicar la transformada de wavelets, a señales procedentes de muestras de acero inoxidable 304 en sus primeras horas de inmersión en disolución de $\mathrm{FeCl}_{3}$ 0,001M, ha sido posible detectar la existencia de dos componentes: una procedente de un proceso de corrosión localizada y otra de uno uniforme. No obstante, habría que tener en cuenta que la detección de diferentes procesos que actúen simultáneamente será posible sólo si se manifiestan en los ENR a una escala temporal lo suficientemente distinta. En estos casos, haciendo uso de los diagramas de distribución de energía sería posible detectar la existencia de estos dos procesos y caracterizarlos en función del valor de la constante de tiempo asociada a los tránsitos que producen en los registros experimentales. Por otra parte, estos diagramas obtenidos para registros de ruido en corriente ofrecen la posibilidad de cuantificar la intensidad relativa de los procesos. Todo ello podría dar lugar a un sistema automático para el seguimiento de procesos de corrosión en el que se pudiera evaluar si existe más de un proceso de corrosión operando paralelamente y qué evolución presentan con el tiempo. Este tipo de análisis no podría ser llevado a cabo aplicando métodos clásicos de análisis de ENR.

Por otra parte, cuando existen procesos que producen tránsitos aislados en los registros experimentales, se abre la posibilidad de automatizar la obtención de otro tipo de información como es el recuento de tránsitos en un registro experimental, así como su localización en el tiempo para estudiar la pauta de aparición de los tránsitos. Además, estos tránsitos podrían ser clasificados dependiendo de su constante de tiempo.

En resumen, los resultados obtenidos ponen de manifiesto la potencial utilidad de la transformada de wavelets para el análisis de datos de ruido electroquímico. Su empleo permitiría realizar tareas no abordables cuando se utilizan otros métodos de análisis descritos en la bibliografía. Esto permitiría abrir el campo de aplicación de la técnica de ruido electroquímico. No obstante, la dimensión real de la utilidad práctica del método propuesto sólo podrá ser evaluada aplicándolo a un mayor número de sistemas. 


\section{Agradecimientos}

Este trabajo ha sido financiado gracias a las ayudas concedidas por la CICYT (Proj. Ref.: MAT-971075-C03-01) y por la Junta de Andalucía.

\section{REFERENCIAS}

[1] A. LEGAT y V. DoleCEK, J. Electrochem. Soc. 142 (1995) 1851-1858.

[2] C. LIU, D.D. MACDONALD, E. MEDINA, J.J. VILLA Y J.M. Bueno, Corrosion 50 (1994) 687-694.

[3] F. MANSFELD y H. XIAO, J. Electrochem. Soc. 140 (1993) 2205-2209.

[4] D.L. REICHERT, Electrochemical Noise Measurement for Corrosion Applications, Ed. ASTM. West Conshohocken, EE.UU., 1996, pp. 79-89.

[5] A. LEGAT, y V. DoleCEK, Corrosion 51 (1995) 295-300.
[6] C. Monticell, F. Zucchi, G. Brunoro y G. Trabanell, J. App. Electrochem. 27 (1997) 325-334.

[7] U. Bertoccl, C. Gabrielu, F. Huet, y M. Keddam, , J. Electrochem. Soc. 144 (1997) 31-43.

[8] T.T. LUNT, S.T. PRIDE, J.R. SCULIY, J.L. HUDSON y A.S. MIKHAllOV, J. Electrochem. Soc. 144 (1997) 16201629.

[9] P.R. ROBERGE, Electrochemical Noise Measurement for Corrosion Applications, Ed. West Conshohocken, EE.UU., 1996, pp. 142-156.

[10] A. Aballe, M. Bethencourt, F.J. Botana, M. Marcos, Electrochim. Acta 44 (1999) 4805-4816.

[11] A. BRUCE y H-Y. GAO, Applied Wavelet Analysis with SPlus, Ed. Springer, New York, EE. UU., 1996.

[12] B.B. HUBBARD, The world according to wavelets, Ed. Peters, AK, Wellesley, EE.UU., 1996.

[13] A. Cohen, I. Daubechies y P. Vial, App. Comp. Harmonic Analysis, 1 (1993) 54-81.

[14] I. DAUBECHIES, Ten lecturers on wavelets, Society for industrial and applied mathematics, Philadelphia, PA, EE.UU., 1992.

[15] D.E. Willams, J. Stewart y P.H. Balkwill, Corros. Sci. 36 (1994) 1213-1235 